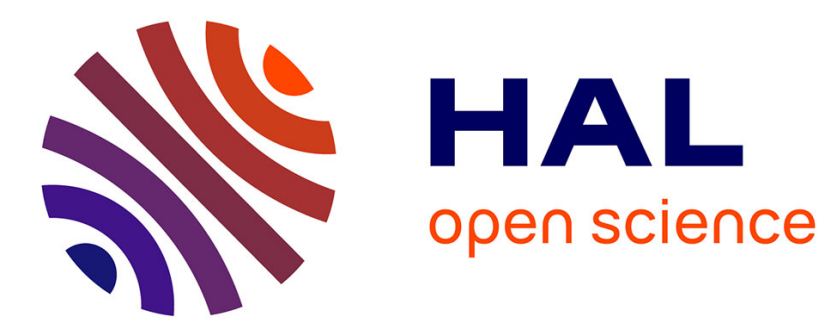

\title{
Structural instability of transition metals upon ultrafast laser irradiation
}

\author{
L. Ben-Mahfoud, E. Silaeva, R. Stoian, Jean-Philippe Colombier
}

\section{To cite this version:}

L. Ben-Mahfoud, E. Silaeva, R. Stoian, Jean-Philippe Colombier. Structural instability of transition metals upon ultrafast laser irradiation. Physical Review B, 2021, 104 (10), 10.1103/PhysRevB.104.104104 . ujm-03340527

\section{HAL Id: ujm-03340527 \\ https://hal-ujm.archives-ouvertes.fr/ujm-03340527}

Submitted on 13 Oct 2021

HAL is a multi-disciplinary open access archive for the deposit and dissemination of scientific research documents, whether they are published or not. The documents may come from teaching and research institutions in France or abroad, or from public or private research centers.
L'archive ouverte pluridisciplinaire HAL, est destinée au dépôt et à la diffusion de documents scientifiques de niveau recherche, publiés ou non, émanant des établissements d'enseignement et de recherche français ou étrangers, des laboratoires publics ou privés. 


\title{
Structural instability of transition metals upon ultrafast laser irradiation
}

\author{
L. Ben-Mahfoud, ${ }^{1}$ E. P. Silaeva, ${ }^{1}$ R. Stoian, ${ }^{1}$ and J.P. Colombier ${ }^{1, *}$ \\ ${ }^{1}$ Univ Lyon, UJM-Saint-Etienne, CNRS, IOGS, Laboratoire Hubert Curien UMR5516, F-42023 St-Etienne, France
}

( Dated: July 7, 2021)

\begin{abstract}
Precision in laser material structuring is critically defined by the energy flow during the irradiation process, particularly in ultrafast regimes. Alternative to thermal evolutions, nonequilibrium electronic excitation can exercise a direct influence on the atomic bonding delivering a potentially rapid destructuring process. In this context, the atomic disordering of transition metals $(\mathrm{Cr}, \mathrm{Ni}$ and Ti) induced by non-equilibrium electronic excitation typical for ultrafast laser processing is studied with an emphasis on the role of the d-band filling and crystalline structure. The density functional theory is used to obtain, from first principles, structural stability criteria and non-thermal disordering pathways on timescales shorter than picosecond electron-phonon dynamics. We show that the hot electrons distort charge distribution in the cold crystalline arrangement and increase the entropy of the system thus driving the mechanical expansion. The calculated nonequilibrium freeenergy potentials indicate that a solid destabilization is possible when electron temperature reaches a universal value of around $2 \mathrm{eV}$ for all considered metals. Under a uniaxial lattice relaxation expected in the laser ablation of surface layers the charge redistribution and loss of lattice stability is shown to be oriented along specific crystal directions. Moreover, we show that the interatomic potential destabilization is energetically more favorable for $\mathrm{Ni}$ than for $\mathrm{Cr}$ and $\mathrm{Ti}$. Lattice dynamics is affected by space charge redistribution induced by Fermi smearing associated with anisotropic expansion due to electron pressure gradients. This ultrafast destructuring mechanism is shown to be a general feature of partially-filled-d-band transition metals.
\end{abstract}

\section{INTRODUCTION}

Ultrafast laser surface processing proceeds commonly via material ablation, where the localized energy deposited in the solid is sufficient to initiate material ejection [1. The time required for this transformation and the amount of energy left in the solid define the spatial limits of laser processing and its resolution with respect to the optical size of the beam. Assuming ultrafast electronic excitation on the timescale of the laser pulse, the thermal scenarios imply picosecond energy transfer to the phonons and vibrational degrees of freedom, gradually heating the solid beyond the mechanical stability limit [2, 3]. A faster transformation process shortens the energy diffusion, contributing to higher precision and reduced collateral damage. This intrinsically implies an electronically-driven destructuring pathway. An ultrafast non-collisional coupling between photons, electrons and matrix can lead to coherent spin polarization and lattice dynamics at low-dose excitation [4 6] and to ultrafast phase transitions and ablation at high doses [7, 8].

The emergence of non-thermal transformation pathways was discussed extensively for covalent materials following the early scenarios for semiconductor annealing 9 11]. Their stability becomes under strain as the electrons are optically excited above a critical value. If few percent of the valence band electrons are pumped in the conduction band antibonding states, molecular potentials flatten, transverse acoustic phonons become unstable, triggering the collapse of the whole structure on a $100 \mathrm{fs}$ timescale and initiating a non-thermal melting of

\footnotetext{
* jean.philippe.colombier@univ-st-etienne.fr
}

the crystal 12 14. The non-thermal character was implicitly linked to the speed of transformation, occurring faster than the electron-phonon equilibration time, meaning at the same time that energy is transferred to the lattice as potential deformation energy and not collisionally via gradual phonon activation at the typical energies of several meV. The experimental evidence from ultrafast structural probes [15 confirms the rapid structural transition towards disordered states resembling a melt. First comprehensive disordering scenarios described a selective flattening of atomic potentials and softening of the direction-specific acoustic modes, particularly branches of transverse modes in the Brillouin zone [12, 16, 17] with increasing anharmonicity. Alternative approaches suggested the overall barrierless potential energy surfaces driving inertial, unrestricted dynamics of the atoms [15]. This view was challenged by the acoustic phonon models, indicating that the softening of few acoustic modes may be sufficient to trigger the whole matrix collapse [17. The process can be effectively enhanced by the swift excitation of optical phonon modes if available, resulting in displacive relaxation and the excitation of vibrational amplitude above the Lindemann limit [18. This limit usually serves as a disordering structural criterion and the time evolution of the average atomic displacements is needed to unambiguously asses melting [19, 20. Bismuth Peierls-distorted crystal lattice [21, 22] and mixed ionic-covalent bonded solids [23, 24] are other types of crystalline solids concerned by non-thermal lattice softening.

The concept of ultrafast disordering is less common for pure metals due to an expected fast electronic screening of the laser-induced charge non-equilibrium, with the prevalence of thermal models for melting. A non- 
equilibrium is mostly considered for the distribution of hot electrons as well as, more recently, for the distribution of the phonons. Their evolution away from the respective Fermi-Dirac and Bose-Einstein functions will affect electron-phonon coupling and the speed of energy transfer [25, 26]. Non-equilibrium electronic, optical, and thermal properties of excited metals were calculated from electronic temperature dependent first-principles theories 27, 28, and, in general, the thermophysical properties evolve according to the coupling between electronic and molecular degrees of freedom [25, 27 27 35]. Whether electronic excitation can speed up laser induced melting is still an open question [36 38. The possibility to trigger phase transitions in a non-thermal way has been advocated, for example in a prototype of a freeelectron like metal $(\mathrm{Al})$ following interband excitation [39, a view challenged later by first-principles calculations showing that electronic heating in $\mathrm{Al}$ produces no significant changes in the electronic structure and therefore not a significant drive for lattice dynamics [40]. The preparation of homogeneous warm-dense-matter conditions in gold, a full $d$-band model, probed by ultrafast X-ray absorption sensitive to electronic temperature 41. indicate that thermal transitions may occur in nonequilibrium conditions (i.e. before electron-phonon equilibration) in about $1 \mathrm{ps}$, still in the limits of a two-temperature model, with structural tools sensitive to ionic arrangements showing a slower melting process [42]. However for transition metals, the electronic excitation seems to influence the lattice stability [40,43]. The question of stability was raised from a first-principles perspective and accompanied by experiments using structural probes. Assuming isochoric heating, Recoules et. al 40 suggested a potential hardening of phonon modes, interpreted as a transient increase of the melting threshold, and observed as an apparent delay in the onset of the liquid by ultrafast diffraction by Ernstorfer et al. [44. This tends to stabilize the metallic lattice, through screening effects via a change of the electronic space charge distribution 33. This scenario can be applied to a large range of metals, except for $\mathrm{W}$ which exhibits phonon instabilities for a certain range of electronic temperatures between 1.7 and $4.3 \mathrm{eV}$ [45. If the isochoric heating is assumed for a homogeneous excitation processes with a characteristic length given by the skin depth, the superficial layers may behave differently undergoing a potentially fast pressure relaxation. This behavior is further stressed by inhomogeneous temperature distributions and strong axial gradients, with unidirectional relaxation. The concept of a rapid material destructuring due to a thermomechanical effect of electron heating was discussed for a transition metal, W, with a driving factor identified in the entropic electronic contribution [46]. Zhang et al. also showed using hydrodynamic simulations that fast transition times can be obtained assuming classical phase transitions driven by ionic temperatures, without implying a complete electron-phonon equilibration [47. The above discussion demonstrates that non-equilibrium processes and paths of material transformation depend on the existing constraints and environmental conditions. Understanding the paths induced by primary excitation events is thus of interest. For example, interatomic potentials revealing a loss of equilibrium position can lead to the lattice destabilization if the change of volume is allowed 46, 48, 49. Solid expansion leads systematically to the phonon softening [43, 50]. Until now, homogenous relaxation has been considered in the study of lattice dynamics with phonon dispersion curves calculated for the equilibrium volume at given electron temperature $V_{e q}\left(T_{e}\right)$ [43, 45]. It is of interest however for surface ablation of transition metals to investigate the possibility to trigger dynamical non-thermal phase transitions under uniaxial lattice relaxations. We therefore propose a working hypothesis consisting in the possibility to trigger an ultrafast destabilization of the cold lattice under a strong electronic heating and uniaxial relaxation.

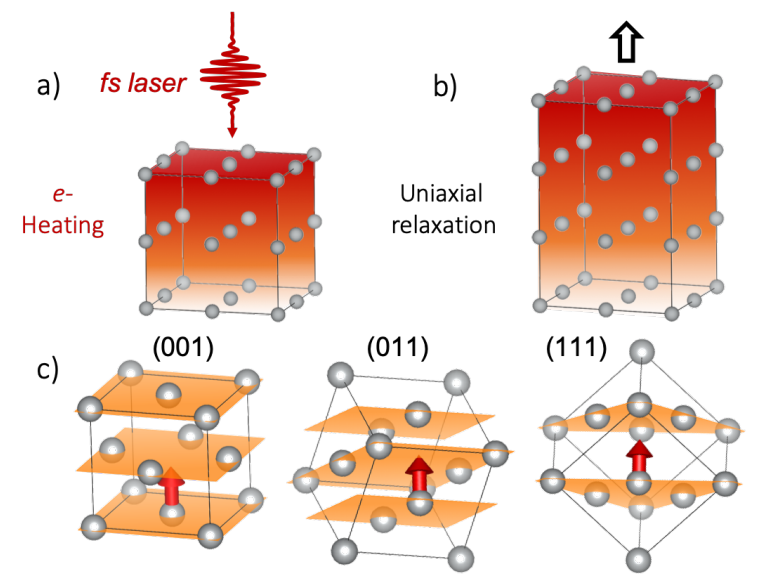

Figure 1. Ultrashort laser-induced heating of the electron population in the photoexcited metal (a). Uniaxial expansion normal to the surface (b) induces lattice relaxation depending on the local crystal surface orientation (c).

In the present manuscript, using first-principles calculations, we discuss the possibility of ultrafast phase transformations in nonequilibrium electron-phonon conditions for transition metals. We consider different crystalline lattice structures: body-centered cubic (bcc) Chromium, face-centered cubic (fcc) Nickel and hexagonal closepacked (hcp) Titanium and orientations: $\mathrm{Cr}$ (001), Ti (001) and $\mathrm{Ni}$ (001), (011) and (111). For these metals the presence of $d$ electrons and the degree of filling of the $d$ band play an important role in non-thermal changes of the electronic and lattice structures. Section II presents the calculation details and the physical assumptions. Section III is devoted to the study of interatomic potentials under nonequilibrium. In Section IV, electronic space charge redistribution along high-symmetry directions is analyzed. The potential lattice dynamics is discussed using the phonon dispersion curves under uniaxial solid expansion in Sec. V. Finally, the results are 
summarized in Sec. VI. Our results indicate the existence of preferential crystalline orientations for inducing dynamical instabilities along uniaxial strain under high electronic temperature. Thereby, it provides an original view on a transient solid state under stress and emerging blast forces induced by electronic disorder.

\section{CALCULATION DETAILS}

Modeling of $\mathrm{Cr}, \mathrm{Ni}$, and $\mathrm{Ti}$ metals under electronphonon nonequilibrium is performed with the code Abinit based on plane-waves description of the electronic wavefunctions 51. Calculations are carried out within the density functional theory (DFT) [52, 53] extended to finite electronic temperatures [54. The generalized gradient approximation (GGA) exchange-correlation functional of Perdew-Burke-Ernzerhof (PBE) 55 with a norm-conserving pseudopotential method is used to determine the relaxed electronic band structures. Ground state valence electrons occupy the $3 \mathrm{~d}$ and $4 \mathrm{~s}$ orbitals in the electronic configuration given by the TrouillerMartins pseudopotentials for the given transition metals [56]. Cr has a typical bbc structure (space group $\operatorname{Im} \overline{3} m$, 229), Ni has a fcc structure (space group $F m \overline{3} m, 225$ ) and Ti has a hcp structure (space group $\left.P 6_{3} / m m c, 194\right)$. $\mathrm{Cr}$ and Ti belong to the same group of transition metals as they both have a partially-filled $d$ band, while $\mathrm{Ni}$ is a nearly-filled- $d$-band transition metal. Plane-wave kinetic energy cutoff of the valence pseudo-wave functions is set to $40 \mathrm{Ha}$. Basic ground state structural properties as lattice constants and bulk modulus, calculated at ambient electronic conditions with $T_{e} \approx 300 \mathrm{~K}$ are in good agreement with experimental values and are summed up in Table I]

In the theoretical description of the irradiated material, we assume that the ultrashort laser interaction induces an instantaneous heating of the electrons whereas the lattice remains undisturbed at $T_{l}=300 \mathrm{~K}$. The intensity of the photoexcitation is emulated by an electronic temperature describing the degree of electron-phonon nonequilbrium, assuming that all sub-shells are thermalized with each other and have not yet transferred their energy into the lattice [57]. The electron sub-system thermalized at $T_{e}$ is then described by a Fermi-Dirac occupation function :

$$
f_{i}=\frac{1}{e^{\left(\epsilon_{i}-\mu_{e}\right) / k_{B} T_{e}}+1}
$$

where $\epsilon_{i}$ are the Kohn-Sham energy eigenvalues, $\mu_{e}$ is the electron chemical potential and $k_{B}$ is the Boltzmann constant. In the Mermin framework [54, the ab initio ground state determination requires the minimization of the electronic free energy, $F_{e}=E-T_{e} S_{e}$, where $E$ is the electronic internal energy and $S_{e}$ the electronic entropy defined as follows for Kohn-Sham independent electrons:

$$
S_{e}=-k_{B} \sum_{i=1}^{+\infty}\left[f_{i} \ln f_{i}+\left(1-f_{i}\right) \ln \left(1-f_{i}\right)\right]
$$

This means that relaxation of the band structure relies on an intricate combination of $E$ minimization and $S_{e}$ maximization.

Geometrical configuration emulating the typical laser surface experiment is presented in Fig. 1. The longitudinal gradient of the electronic pressure $P_{e}$, induced by the laser absorption with its evanescent field leading to electron temperature increase (Fig. 19), gives rise to entropic blast forces. They will foster a potential lattice expansion once constraints are released. Moreover, in an ablation process where a spot size, typically of $50 \mu \mathrm{m}$ diameter on the surface, is significantly larger than the energy deposition depth defined by the optical penetration depth $\delta_{o p t} \simeq 10 \mathrm{~nm}$ and the electronic thermal diffusion length $\delta_{\text {diff }} \simeq 100 \mathrm{~nm}$. Hydrodynamic relaxation in response to the strong longitudinal gradients proceeds perpendicular to the surface as shown in Fig. 1 p. This generates a unidirectional lattice expansion considered in this investigation.

Asymmetric deformation of different crystalline lattice structures has been considered theoretically (Fig. 11.). Together with energy minimization, the dynamic stability of the bcc, fcc and hcp phases is investigated within the density functional perturbation approach (DFPT). Computations are performed at the theoretical equilibrium lattice parameter of the crystal phase obtained at $T_{l}=0 \mathrm{~K}$. In order to sample the Brillouin zone by the Monkhorst-Pack method [58], a $8 \times 8 \times 8$ k-point grid in the reciprocal space is considered for fcc $\mathrm{Ni}$ (001), $9 \times 12 \times 12$ for $\mathrm{Ni}(110)$ and $6 \times 10 \times 4$ for $\mathrm{Ni}(111)$. To obtain the phonon dispersion curves by the calculation of the dynamical matrices, a q-point grid of $2 \times 2 \times 2$ was used for $\mathrm{Ni}(001), 3 \times 4 \times 4$ for $\mathrm{Ni}(110)$ and $3 \times 5 \times 2$ for Ni (110). For Cr (bcc) and Ti (hcp), the reciprocal and phonon wave vector meshes of $16 \times 16 \times 9$ and $4 \times 4 \times 3$ were considered. At the highest temperature $\left(T_{e} \approx 2\right.$ $\mathrm{eV}), 15$ bands per atom were considered to reproduce the effective electron occupations. The convergence of the results was ensured with respect to the cutoff energy, k-point and q-point grids.

To take into account the asymmetric expansion preferentially oriented normal to the irradiated surface and the subsequent inhomogeneous response of crystal grains on a real surface, three different crystal orientations were investigated for each metal. For fcc Ni, (001), (011) and (111) crystal planes were considered. To apply the relaxation along the [001] direction, the conventional unit cell is deformed by increasing the length of the corresponding vector component. To reproduce the strains along the [011] \& [111] directions, a transformation matrix $M$ 


\begin{tabular}{|c|c|c|c|c|c|c|c|c|c|}
\hline Elt & $\mathrm{XC}$ functional & $e^{-}$structure & crystal structure & $a_{t h}$ & $a_{\text {exp }}$ & Rel.err & $\mathrm{B}_{t h}$ & $\mathrm{~B}_{\exp }$ & Rel.err \\
\hline $\mathrm{Cr}$ & GGA & $3 d^{5} 4 s^{1}$ & bcc & 2.89 & 2.91 & $0.69 \%$ & 245 & 279 & $12 \%$ \\
\hline $\mathrm{Ni}$ & GGA & $3 d^{8} 4 s^{2}$ & fcc & 3.54 & 3.52 & $0.57 \%$ & 200 & 180 & $11 \%$ \\
\hline \multirow[t]{2}{*}{$\mathrm{Ti}$} & GGA & $3 d^{2} 4 s^{2}$ & $\mathrm{hcp}(\mathrm{a}, \mathrm{b})$ & 2.93 & 2.95 & $0.68 \%$ & 111 & 116 & $4.3 \%$ \\
\hline & & & hcp(c) & 4.7 & 4.68 & $0.43 \%$ & & & \\
\hline
\end{tabular}

Table I. DFT and experimental values (and their relative errors) of the lattice constants $a$ (in $\AA$ ) and bulk modulus $B$ (in GPa) for three pure transition metals.

has been applied to the crystal orientation as follows:

$$
M_{110}^{f c c}=\left(\begin{array}{ccc}
1 & 1 & 1 \\
-2 & 0 & 1 \\
1 & -1 & 1
\end{array}\right) M_{111}^{f c c}=\left(\begin{array}{ccc}
-0.5 & 0.5 & -1 \\
1 & 0 & -1 \\
-0.5 & -0.5 & -1
\end{array}\right)
$$

This way, the stress relaxation direction in the initial basis corresponds to the [001] direction in the transformed one. The strain is thus effective as we increase the third lattice vector component in the new lattice basis. Next, we present how the ultrafast laser thermal excitation affects the interatomic potential and modifies material stability and dynamics.

\section{NON-EQUILIBRIUM INTERATOMIC POTENTIALS}

In order to evaluate how the electronic temperature affects the lattice cohesion and might induce a fast lattice destabilization toward non-crystal phases without phonon mediation, static interatomic potentials are investigated. They are obtained by calculating the total energy of the metallic system at different interatomic separations considering an uniaxial relaxation mechanism perpendicular to the surface for different degrees of photoexcitation corresponding to several values of electronic temperatures. As expected, at ambient conditions $\left(T_{e}=\right.$ $0.026 \mathrm{eV})$, the metal lattice is stable and the interatomic potentials shown in Fig. 2 exhibit a minimum at the equilibrium lattice parameter $a_{0}$. Moreover, the local curvature of the potentials around this equilibrium position defines the theoretical bulk moduli which are in good agreement with the experimental values as shown in Table I

The calculations at higher electronic temperatures show that the interatomic potential minima are shifted with $T_{e}$ to the higher interatomic distance (not shown). This behavior is expected and can be attributed to an adaptation of the system to the build-up of electronic pressure. More remarkably, the increase of $T_{e}$ reduces the curvature of the potential. The curve flattening indicates the loss of the free energy minimum in agreement with the previous reports on homogeneous relaxation [46, 48, 49]. This occurs for an electronic temperature around $2 \mathrm{eV}$ for all considered metals, as shown in Fig. 2. Particularly, for each material the thresholds are $T_{e}^{t h}(\mathrm{Cr})=2.15 \mathrm{eV}, T_{e}^{t h}(\mathrm{Ni})=2 \mathrm{eV}, T_{e}^{t h}(\mathrm{Ti})=$
$2.05 \mathrm{eV}$. Above this stability threshold, the lattice structure is no longer energetically stable. A similar stability threshold was determined for Tantalum under homogeneous expansion $\left(T_{e}^{t h}(\mathrm{Ta})=25000 \mathrm{~K} \approx 2.15 \mathrm{eV}\right)$ [59]. Thereby, $T_{e} \simeq 2 \mathrm{eV}$ seems to be a universal value for all transition metals. The loss of the energy minimum corresponding to the equilibrium distance results from the dominant entropic contribution as the electronic disorder increases with $T_{e}$ [47, 48. This is directly related to the volume expansion of the phase space available for the electrons. This contribution is visible in the evolution of the free energy of the electronic system.

The energy $\varepsilon_{e}^{t h}$ required to reach the threshold electronic temperature depends on the material electronic heat capacity as $\varepsilon_{e}^{t h}=\int_{0}^{T_{e}^{t h}} C_{e} d T_{e}$. For the considered metals, these energy values range from $3 \mathrm{eV} /$ atom to more than $5 \mathrm{eV} /$ atom. They are summarized in Table II and compared with the vaporization heat induced by classic thermal mechanisms and the experimental cohesive energy reflecting the bond strength. We notice

\begin{tabular}{cccc}
\hline \hline & $\begin{array}{c}\text { Nonthermal } \\
\text { destructuring } \\
\text { energy (eV/atom) }\end{array}$ & $\begin{array}{c}\text { Vaporization } \\
\text { heat } \\
(\mathrm{eV} / \text { atom })\end{array}$ & $\begin{array}{c}\text { Cohesive } \\
\text { energy } \\
(\mathrm{eV} / \text { atom })\end{array}$ \\
\hline $\mathrm{Cr}$ & 5.73 & 3.51 & 4.10 \\
$\mathrm{Ni}$ & 3.36 & 4.40 & 4.44 \\
$\mathrm{Ti}$ & 4.61 & 3.91 & 4.85 \\
\hline \hline
\end{tabular}

Table II. Theoretical values of the energy required to induce an energetical destabilization compared with the experimental heat of vaporization and cohesive energy [60].

that for $\mathrm{Ni}$, the lattice destabilization via electronic effects required less energy than needed for thermal ways and even breaking the atomic bonds. The opposite is observed for $\mathrm{Cr}$ and $\mathrm{Ti}$ as the heat of vaporization and cohesive energy are lower than the required energy to destabilize the lattice in a non-thermal way. In other words, the fast way is cheaper energetically for $\mathrm{Ni}$ which could foster an effective destabilization layer through ultrafast electronic photoexcitation, preventing thermal damages. Moreover, Ni is particularly interesting as the electronphonon coupling strength is known to decrease strongly with electronic temperature [25, 29]. This result emphasizes the opportunity to have a longer nonequilibrium state, delaying thermal coupling and giving more time 

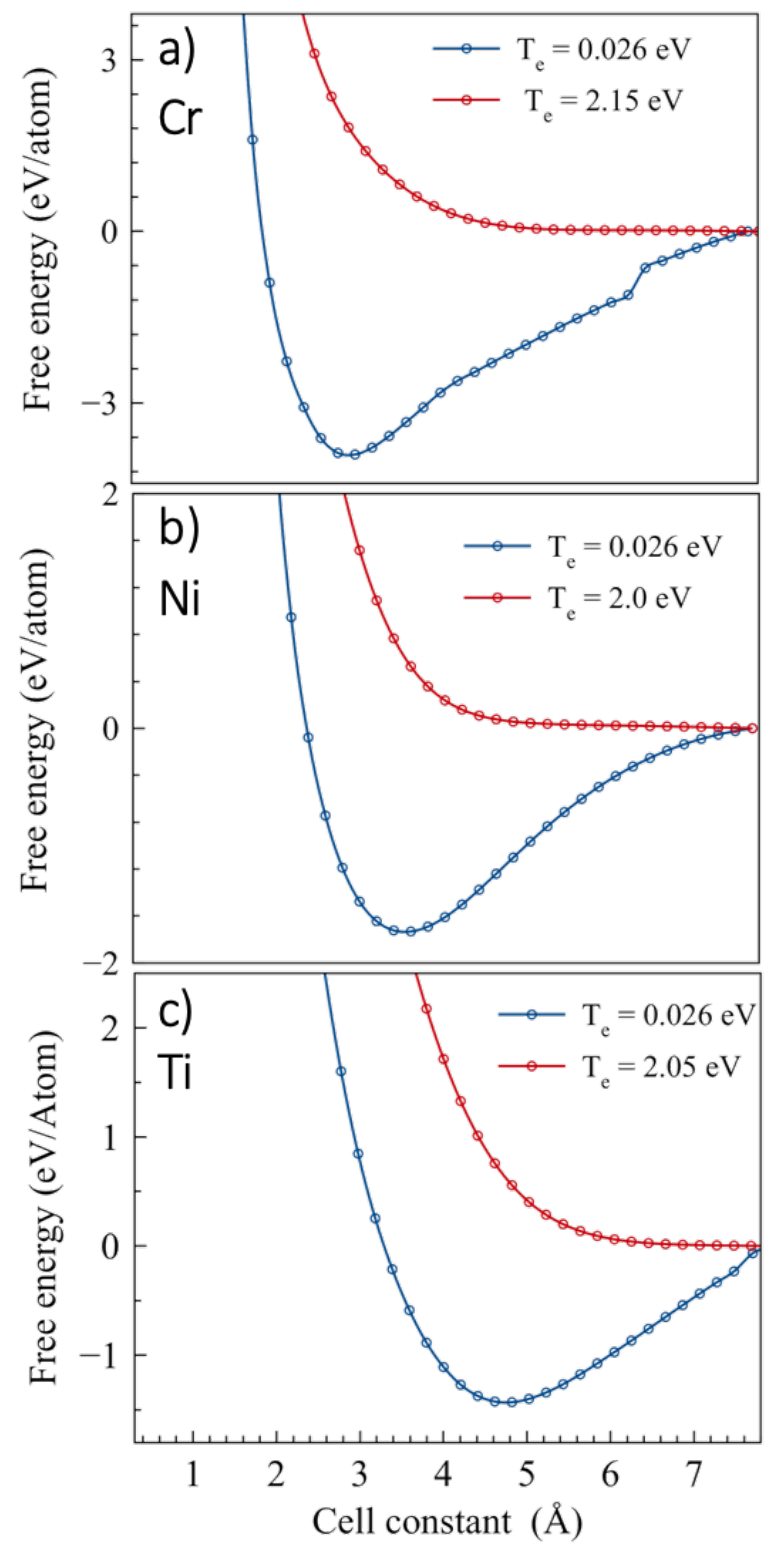

Figure 2. Evolution of the free energy curve as $T_{e}$ is increased from $0.026 \mathrm{eV}$ to the stability threshold. At $T_{e}=0.026 \mathrm{eV}$ the free energy shows a minimum value revealing an equilibrium position. Above the indicated threshold temperature, the disappearance of the free energy minimum indicates a possible loss of stability.

for non-thermal destabilization to occur.

The precise band structure drives the volumedependence of the electron states near the Fermi level and determines the entropic value that may exceed the internal energy for distance larger than the equilibrium one. At the same time, a pressure increase in the tens of GPa range at $T_{e}=2 \mathrm{eV}$ establishes the mechanical drive in the optical penetration depth 32. As mentioned by Tanaka et al 48, a pure entropic effect induced by electronic pressure could destabilize the system resulting in non-thermal metal ablation. Nevertheless, in order to get a global view of the destabilization scenario, this static picture is not enough as free energy barrier can prevent spontaneous transformation. Below we complete this investigation by presenting the electronic redistribution followed by the dynamic response of the lattice for electron heating and a uniaxial expansion.

\section{ELECTRONIC RESTRUCTURING}

Upon ultrafast heating, the electrons are rapidly thermalized by electron-electron interaction and simultaneously reorganized with a smearing of the occupation number over initially empty bands. At a fixed electron temperature, the energy states filling follows the Fermi-Dirac distribution with a modified density of states (DOS) reevaluted consistently by free energy minimization. The DOS modification induces a charge redistribution on the attosecond timescale in the unit cell of the crystalline lattice 57, 61. Figure 3 shows the isochoric electronic density difference $\Delta n_{e}(r)$, calculated between two electronic temperatures: $\Delta n_{e}(r)=n_{e}\left(r, T_{e}^{t h}\right)-n_{e}\left(r, T_{e}^{300 K}\right)$, and represented for the isovalues selected for each material to emphasize the local changes.

The change in the electron localization level around the nucleus directly depends on the degree of $d$-band filling. The localization degree increases near the nucleus for partially-filled $d$ band transition metals $\mathrm{Cr}$ and $\mathrm{Ti}$, and decrease for nearly-filled $d$ band metal as $\mathrm{Ni}$. While the $d$ electrons are more localized at higher $T_{e}$, the electron transfer from $d$ to $s p$ band leads to the electron delocalization [43]. For $\mathrm{Cr}$ and $\mathrm{Ti}$, we observe that the increase of electron temperature induces a charge space relocalization around the nucleus, which has been previously associated with electronic screening [32]. The $n_{e}$ redistribution impacts the ion screening and subsequent interatomic forces. For this kind of transition metals, $T_{e}$ increase induces an increase of the Hartree energy associated to a moderate strengthening of the localization degree for the $d$ band electrons. Nuclei also tend to attract $s p$-band free electrons leading to a loss of delocalized electron distribution (observed at lower electron density isovalues not shown here). For Ni, the electrons become more delocalized leaving the $d$ band to join the $s p$ states as the Hartree energy becomes more negative with the increase of $T_{e}$ 32. This inhomogeneous relocalization observed in Fig. 3. (b) is in agreement with previously reported results on other fcc nearly-filled- $d$-band metals 43, 47.

Remarkably, an important electronic gain is visible i Fig. 3(a) in the [001] direction for Cr. This can be generalized to most bcc metals with $\mathrm{W}$ showing a similar behaviour [45, 47]. For hcp Ti, on the contrary, electronic density decreases strongly along the main axis [001] as shown in Fig. 3(c). For Ni, a loss of electronic density is observed along the [001] longitudinal direction and an electronic density gain around nuclei along [011] corre- 
a)

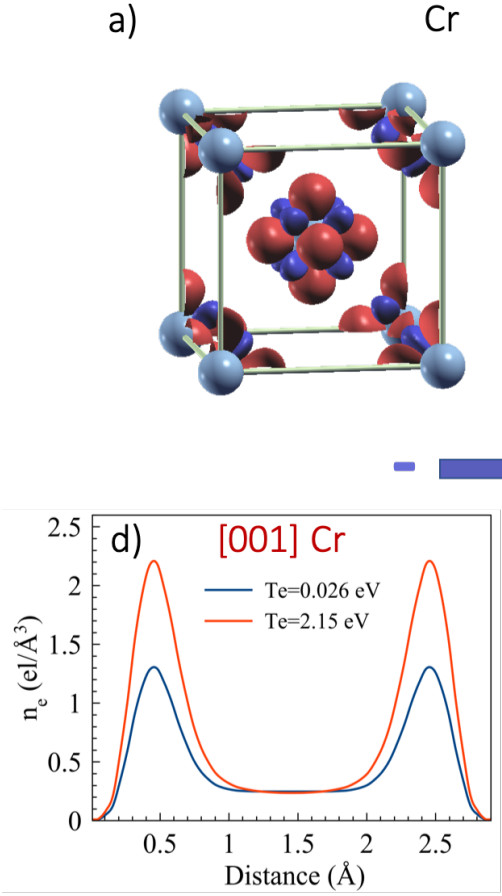

b)

$\mathrm{Ni}$

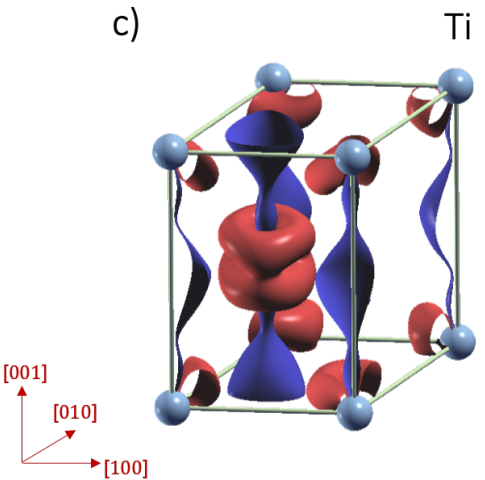

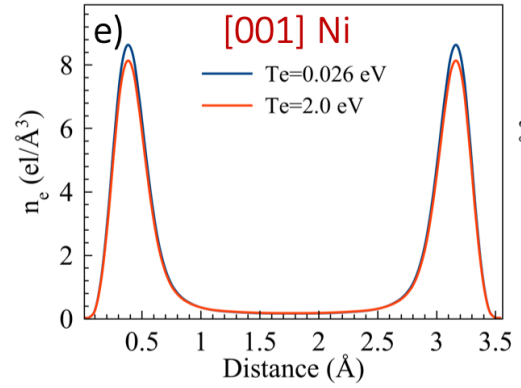

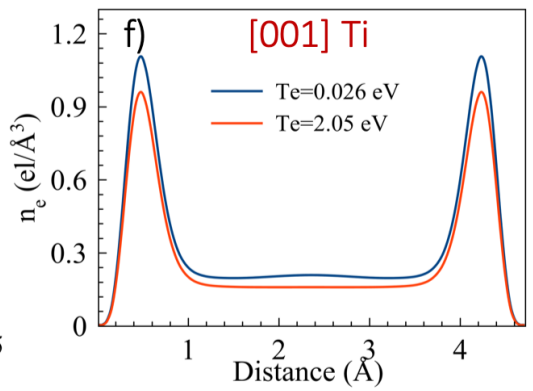

Figure 3. Electronic charge redistribution $\Delta n_{e}(r)=n_{e}\left(r, T_{e}^{t h}\right)-n_{e}\left(r, T_{e}^{300 K}\right)$ for $\mathrm{Cr}(\mathrm{a})$, Ni (b) and Ti (c) for electron density isovalues equal to $0.13 \AA^{-3}, 0.067 \AA^{-3}$ and $0.034 \AA^{-3}$ respectively. Loss and gain of electrons are represented in blue and red, respectively. Electronic density profiles along the [001] directions of the lattice cell for $\mathrm{Cr}$ (d), $\mathrm{Ni}$ (e), $\mathrm{Ti}$ (f) for cold (blue) and hot (red) electrons.

sponding to the short diagonals of the cubic cell represented in cartesian coordinates as shown in Fig. 3(b). Note that for cubic structures, [001] direction is parallel to any axis of the cube whereas, for hcp structure, [001] is associated to the direction of the long lattice constant. A slight $n_{e}$ loss along the [011] direction for $\mathrm{Cr}$ and a slight gain along the [011] and [111] directions for $\mathrm{Ni}$ can be observed but the highest modification of electron density occurs along the [001] direction as shown in Fig. 3(d-f) for $T_{e}=0.026 \mathrm{eV}$ and $T_{e} \simeq 2 \mathrm{eV}$. An important gain of electronic density is revealed for $\mathrm{Cr}$ and only a slight loss for $\mathrm{Ni}$ and $\mathrm{Ti}$ (Fig. $3(\mathrm{~d}-\mathrm{f})$ ). This suggests that the mechanical response to an uniaxial relaxation (corresponding to the shock release geometry upon laser-surface excitation) could be strongly dependent both on the crystal orientation and lattice arrangement. The laser-induced expansion is supposed to apply an outward pressure normal to the surface that may overcome the $d$-electron negative partial pressure and detach the atomic surface planes from underlying bulk 62 . The $d$ electron contribution to the bonding is short-range but is different for the considered metals. The lattice dynamics for thermally excited metals is investigated hereafter for various irradiation configurations.

\section{LATTICE DYNAMICS}

For irradiated materials, DFT revealed that the isochoric increase of electronic temperature induces a hardening of most of the phonon branches in transition metals [40, 63. In particular, with volume fixed at its ambient condition equilibrium value $\left(V_{e q}(300 K)\right)$, the bond hardening appears above an electronic temperature threshold $T_{e}=25000 \mathrm{~K}(\approx 2.15 \mathrm{eV})$ for $\mathrm{Au}[43$. While the interatomic potentials reveal a loss of equilibrium position for $T_{e} \approx 2 \mathrm{eV}$, the phonon spectrum on the contrary shows a stability strengthened by the thermal excitation, mainly related to the induced pressure in the electron system. The dynamical softening appears however as the crystal is progressively relaxed to reduce the electronic pressure [47. Phonon spectra calculated at $V_{e q}\left(T_{e}\right)$ exhibit phonon softening for all electronic temperatures [43] with the solid expansion leading systematically to a dynamical softening of the lattice structure. However, this isotropic relaxation is irrelevant in the context of laser-surface interaction where, upon the electron pressure build-up, the lattice can only relax along the direction perpendicular to the surface.

To assess whether a particular structural arrangement is dynamically stable under uniaxial relaxation, the [001] direction has been chosen because electronic density distribution was shown to be strongly impacted by the electronic heating, with crystal structure specificities. We 

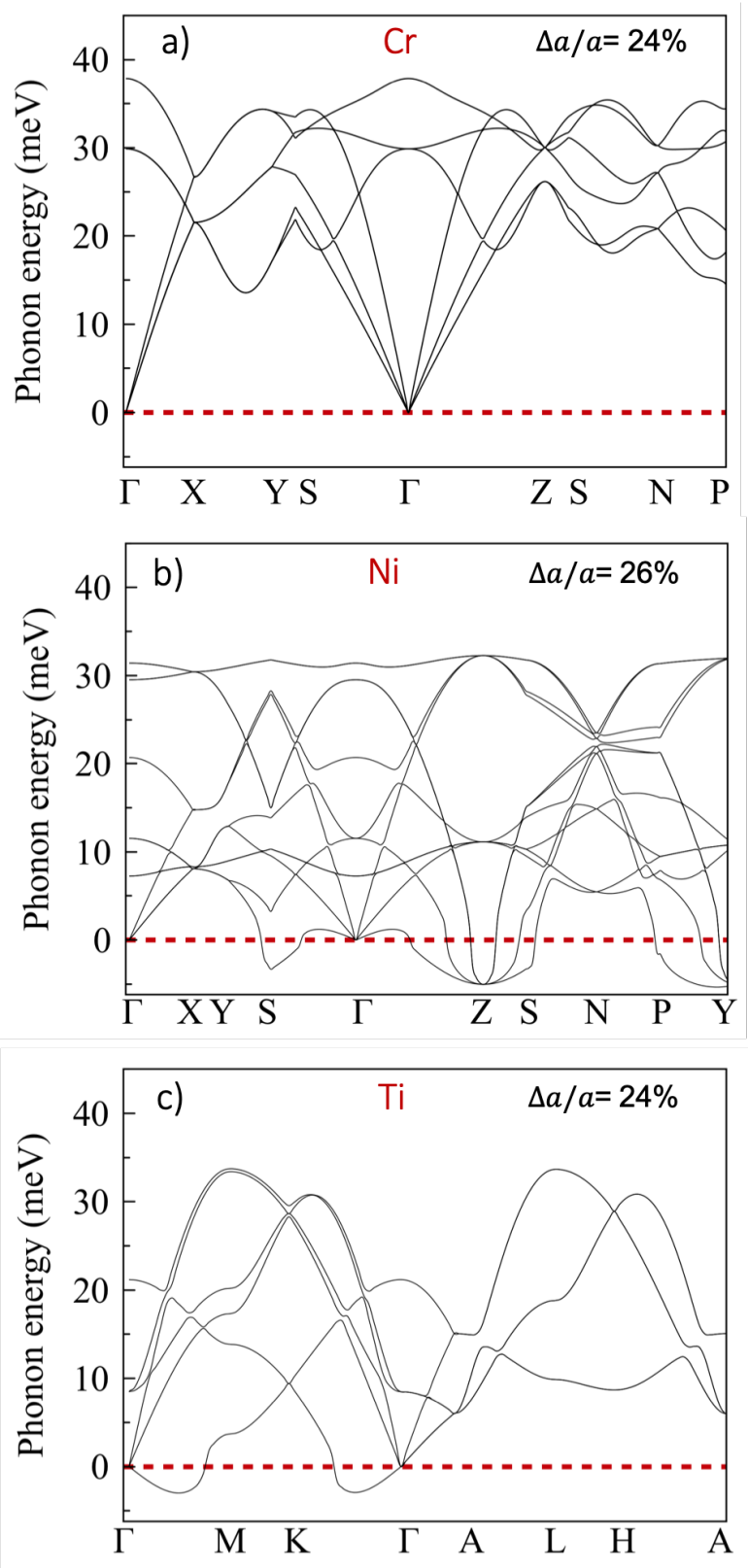

Figure 4. Phonon spectra for a lattice relaxation $\Delta a / a$ near the onset of instabilities along the [001] direction for $\mathrm{Cr}$ (a), $\mathrm{Ni}(\mathrm{b})$ and $\mathrm{Ti}(\mathrm{c})$ at an electronic temperature of $2 \mathrm{eV}$.

have calculated the phonon dispersion curves by DFPT for a crystal structure relaxed along the [001] direction with electrons heated up to the threshold $T_{e} \simeq 2 \mathrm{eV}$. A partial uniaxial release of $20 \%$ has been applied to still keep an active electronic pressure. This corresponds to an atomic displacement less than $1 \AA$ that can be rapidly achieved in a characteristic timescale of $\tau_{a t} \sim 10-20 \mathrm{fs}$ at the sound velocity. As this time is shorter than the electron-phonon relaxation timescale, the system is found in a hot state under partial mechanical relaxation driven by $\nabla P_{e}$.

The lattice dynamics at a similar strain rate applied in the [001] direction is presented in Fig. 4 for the three considered metals. The lattice structure is deformed with $\mathrm{Ti}$ remaining hexagonal but $\mathrm{Cr}$ and $\mathrm{Ni}$ evolving from a cubic to a tetragonal structure. In the phonon spectrum, dynamical instabilities correspond to localized imaginary frequencies within the Brillouin Zone (BZ). Dynamical instabilities in specific directions of the BZ are commonly referred as a solid-solid transition, while the localization of instabilities for several modes in all the directions of the BZ is associated to a loss of lattice periodicity as an amorphization or a solid-liquid transition 64. The phonon dispersion curves reveal that the $\mathrm{Cr}$ stability is preserved (Fig. 4(a)) while the deformed structures of $\mathrm{Ni}$ and $\mathrm{Ti}$ suffer from phonon instabilities as shown in Fig. 4(b-c). Gradual increase of relaxation shows that Ni is dynamically stable up to $\Delta a / a=20 \%$ and imaginary phonon frequencies start to appear in the PY, ZS and $\Gamma Z$ directions of the $\mathrm{BZ}$ for $\Delta a / a=26 \%$. While most of the phonon branches undergo an expected hardening effects [40, soft modes appear in Ti phonon spectrum in specific directions of the $\mathrm{BZ}$ in the $\Gamma \mathrm{M}$ and $\mathrm{K} \Gamma$ directions and become unstable for $\Delta a / a=24 \%$. As for both metals the observed instabilities are localized in specific directions in the BZ, the relaxation of the nonequilibrium system may induce a solid-solid phase transition or trigger defect formation.

Under [001] deformation, Cr phonon spectrum exhibits a remarkable lattice stability even for high relaxation rates up to $\Delta a / a=60 \%$. This behaviour is similar for $\mathrm{W}$ and may be easily generalized to most of bcc metals. Previous DFPT, 2T-MD and DFT-MD studies shown that excited bcc W can undergo a solid-solid phase transition on a picosecond time scale with sufficient electron heating [45 47. In particular, under a uniaxial expansion, the bcc structure undergoes a martensitic phase transformation along the tetragonal Bain path into the fcc phase, with potential stacking fault formations before melting [46. Results on $\mathrm{Cr}$ confirm that the [001] deformation assists the natural tetragonal transformation of bcc metals induced by electron thermal excitation and pressure.

The phase transitions due to the relaxation along other high-symmetry directions can also be possible. In particular, experimental investigations performed on Ni showed that femtosecond-laser irradiation can induce crystalorientation dependent features, leading to the lattice distortion, defects or nanostructure formation [65, 66]. We have also shown in Sec. III that for $\mathrm{Ni}$ a nonthermal destructuring is energetically more favorable compared to other relaxation processes. Moreover, Ni (100) planes are known to undergo an anomalous thermal expansion associated with large anharmonicity in the interatomic potential being a precursor to surface disordering transitions 62. Finally, in our previous study, the thermodynamic stability of fcc-hcp phases was shown to reverse for $T_{e}>1 \mathrm{eV}$ for Ni but an energy barrier is present and the conditions for dynamic instability was not reached 67. This $d$-band metal thus offers a high potential for inducing a collective displacement of atoms triggering a 

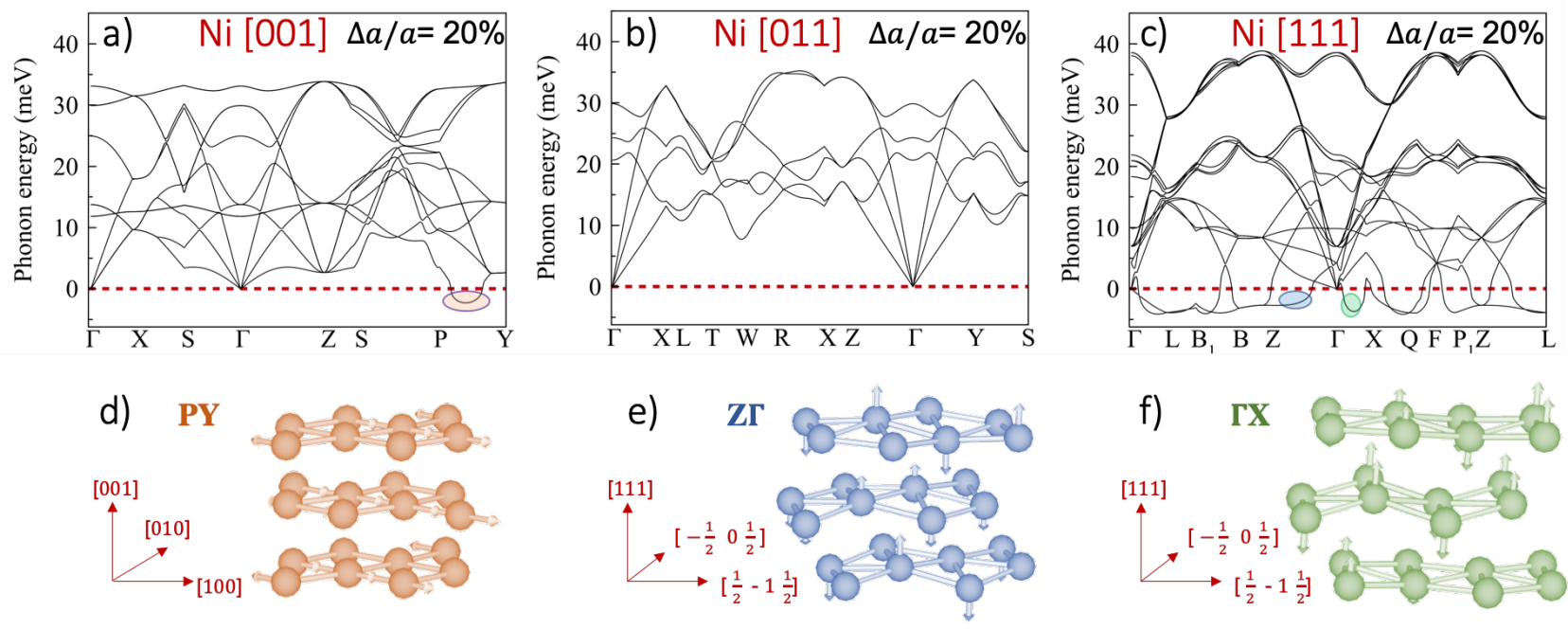

Figure 5. Phonon spectra for a lattice relaxation of $20 \%$ in the [001] (a), [011] (b) and [111] (c) crystal directions for Ni at $T_{e}=2 \mathrm{eV}$ represented in the BZ. Unstable phonon modes along the directions PY for [001] (d), and Z directions for [111] (e)-(f) are illustrated by the atom oscillations in spatial domain.

nonconventional solid-to-solid transformation.

Figure 5 compares the calculated phonon branches under electronic excitation and uniaxial strain along the high-symmetry directions [001], [011] \& [111] for $\mathrm{Ni}$ at $T_{e}=2 \mathrm{eV}$. A relaxation of $\Delta a / a$ has been applied along each directions to activate the weakening of local modes and trigger potential instabilities. During the relaxation, the lattice structure progressively evolves from fcc to tetragonal for $\mathrm{Ni}$ [001], orthorhombic for $\mathrm{Ni}$ [011], and monoclinic for $\mathrm{Ni}$ [111]. As already discussed, [001] relaxation induces the onset of an imaginary frequency for the PY branch observed in Fig. 5a. The stress is anisotropic and the vibration affects the bonding strengths in a plane transverse to the relaxation illustrated by the atom movements of the PY mode shown in Fig. 5 $\mathrm{d}$. Because of the fcc symmetry, the corresponding oscillations remain aligned to [100] direction. For a relaxation along [011] direction, corresponding to an irradiated (011) crystal surface orientation, the phonon spectrum does not suffer from any instabilities (Fig. 5 b). Higher $\Delta a / a$ values also confirmed that the (110) surface has a stiffer holding potential conserving the pristine crystal structure compared to the in-plane bonds. Fig. 5 ; shows the phonon dispersion curves for a relaxation along [111] direction and reveal imaginary frequencies in the whole BZ. This could be a signature of a high disordering capable to induce lattice collapse and a solid-liquid transition before the thermal vibration of the atoms triggers melting according to the Lindemann criterion. These dramatic structural changes, unexpected for the close-packed (111) surface are more likely associated with a mechanical instability, congruent with the Born criterion for melting [68]. Two oscillation modes represented in the spatial domain indicate the atom vibrations along the [111] relaxation direction (Fig. 5p-f). All the unstable branches show a similar directionality with a slight gain of localized $d$ electrons along this orientation. Although this gain seems to be insufficient to prevent the lattice structure collapse.

These results demonstrate that the efficiency of ultrafast non-thermal lattice destructuring upon electronic excitation and unidirectional relaxation depends both on the lattice structure and the surface crystal orientation implying charge space redistribution effects. The strain applied along the [111] direction is identified as the most efficient to destabilize fcc $\mathrm{Ni}$. If the lattice volume has time to adjust to the electronic excitation, it can be possible to experimentally choose the crystal orientation to make the ultrafast destabilization more efficient and to interpret inhomogeneous response of polycrystalline surface materials.

\section{CONCLUSION}

During ultrashort laser-metal interaction, the mechanisms involved in phase transformation determine the rapidity and the precision of the surface structuring process. The ultrafast laser pulse produces an intense electronic excitation that deforms the electronic structure to accommodate the energy of interaction between the electrons. A mechanical drive originates from the entropy gain. This can affect the structural stability of the metal. We have investigated this effect for $\mathrm{Cr}, \mathrm{Ni}$ and $\mathrm{Ti}$ representing three typical crystal structures of transition metals: bcc, fcc and hcp, respectively. A theoretical approach was proposed to assess the relevance of a nonthermal destructuring process on the hundreds of femtosecond timescale, faster than electron-phonon dynamics. On this time scale electronic heating drives the charge redistribution and solid expansion. The lattice destabilization scenario was investigated under electron-phonon nonequilibrium relying on density functional theory and 
density functional perturbation theory. A 3D mapping of the electron gain and loss shows that the degree of $d$-band filling regulates the transfer rate from $d$ localized states to $s p$ delocalized states. The anisotropy in the electron redistribution has been correlated directly with the changes in $d$-band filling. This impacts the electron screening of ions and also induces a direction-dependent electronic stress with entropy increase that can lead to nonthermal phase transition.

The nonequilibrium interatomic potentials under ultrafast heating is shown to exhibit a free energy minimum loss. In a remarkable way, this happens at a temperature threshold $T_{e} \approx 2 \mathrm{eV}$ for all the metals. For $\mathrm{Ni}$ such fast non-thermal loss of stability is energetically cheaper than a thermal path. For $\mathrm{Cr}$ and Ti however this may require more energy. The energetic destabilization is driven by a strong electronic entropy increase that can weaken the lattice cohesion along specific directions. Laser absorption gradients can also give rise to entropic blast forces relaxing the system along only one direction determined by the photoexcited crystal surface orientation. The relaxation of the electronic stress along anisotropic paths matching the weakened lattice vibration directions was thus proposed as a way to foster the surface destabilization pathways. DFPT approach confirms that a dynamical destabilization of the lattice can occur through local mode instabilities in the phonon dispersion curves. In particular, phonon dispersion curve for $T_{e} \approx 2 \mathrm{eV}$ and relaxation along [001] lattice axis differs between the metals. While Cr remains unconditionally stable with strain increase, instabilities appear for some phonon branches in the $\mathrm{BZ}$ of $\mathrm{Ni}$ and $\mathrm{Ti}$. Moreover, we show that [001] and [111] are two preferential crystalline orientations for $\mathrm{Ni}$ where dynamical instabilities can appear under a uniaxial strain and high electronic temperature. This could explain why the surface response of laser-irradiated polycrystals are grain orientation dependent. Finally, we have shown that the lattice vibration of unstable modes were oriented parallel to the [100] direction for [001] relaxation and in the direction of relaxation for the [111] relaxation. The mechanical instabilities driven by electron pressure gradients destabilize the lattice cohesion, in some circumstances fostered by a thermally-induced loss of electronic charge along the relaxation direction. This opens a route for crystal-orientation strategies to destabilize lattice by ultrafast laser irradiation, with a high potential for many nanoscience surface applications.

\section{ACKNOWLEDGEMENTS}

This work was supported by the IMOTEP project within the program "Investissements d'Avenir" operated by ADEME and by the LABEX MANUTECH-SISE (ANR-10-LABX-0075) of the Universite de Lyon, within the program "Investissements d'Avenir" (ANR-11IDEX-0007) operated by the French National Research Agency (ANR). Numerical calculations have been performed using HPC resources from GENCI-TGCC and CINES, project gen7041.
[1] B. N. Chichkov, C. Momma, S. Nolte, F. von Alvensleben, and A. Tünnermann, Femtosecond, picosecond and nanosecond laser ablation of solids, Applied Physics A: Materials Science \& Processing 63, 109 (1996)

[2] J.-P. Colombier, P. Combis, F. Bonneau, R. Le Harzic, and E. Audouard, Hydrodynamic simulations of metal ablation by femtosecond laser irradiation, arXiv e-prints , cond-mat/0612585 (2006), arXiv:cond-mat/0612585 [cond-mat.other],

[3] P. Lorazo, L. J. Lewis, and M. Meunier, Short-Pulse Laser Ablation of Solids: From Phase Explosion to Fragmentation, Phys. Rev. Lett. 91, 225502 (2003)

[4] G. P. Zhang and W. Hübner, Laser-Induced Ultrafast Demagnetization in Ferromagnetic Metals, Physical Review Letters 85, 3025 (2000), publisher: American Physical Society.

[5] J.-Y. Bigot, M. Vomir, and E. Beaurepaire, Coherent ultrafast magnetism induced by femtosecond laser pulses, Nature Physics 5, 515 (2009), number: 7 Publisher: Nature Publishing Group.

[6] C. Rose-Petruck, R. Jimenez, T. Guo, A. Cavalleri, C. W. Siders, F. Rksi, J. A. Squier, B. C. Walker, K. R. Wilson, and C. P. J. Barty, Picosecond-milliångström lattice dynamics measured by ultrafast x-ray diffraction, Nature
398, 310 (1999), number: 6725 Publisher: Nature Publishing Group.

[7] S. Williamson, G. Mourou, and J. C. M. Li, Timeresolved laser-induced phase transformation in aluminum, Physical Review Letters 52, 2364 (1984), publisher: APS.

[8] M. E. Povarnitsyn, K. V. Khishchenko, and P. R. Levashov, Phase transitions in femtosecond laser ablation, Applied Surface Science Laser and Plasma in Micro- and Nano-Scale Materials Processing and Diagnostics, 255, 5120 (2009)

[9] J. A. Van Vechten, R. Tsu, and F. W. Saris, Nonthermal pulsed laser annealing of Si; plasma annealing, Physics Letters A 74, 422 (1979).

[10] R. F. Wood, J. R. Kirkpatrick, and G. E. Giles, Macroscopic theory of pulsed-laser annealing. II. Dopant diffusion and segregation, Phys. Rev. B 23, 5555 (1981).

[11] D. von der Linde and N. Fabricius, Observation of an electronic plasma in picosecond laser annealing of silicon, Applied Physics Letters 41, 991 (1982).

[12] P. Stampfli and K. H. Bennemann, Theory for the instability of the diamond structure of $\mathrm{Si}, \mathrm{Ge}$, and $\mathrm{C}$ induced by a dense electron-hole plasma, Physical Review B 42, 7163 (1990), publisher: American Physical Society.

[13] K. Sokolowski-Tinten, J. Bialkowski, M. Boing, A. Cav- 
alleri, and D. von der Linde, Thermal and nonthermal melting of gallium arsenide after femtosecond laser excitation, Phys. Rev. B 58, R11805 (1998).

[14] M. Harb, R. Ernstorfer, C. T. Hebeisen, G. Sciaini, W. Peng, T. Dartigalongue, M. A. Eriksson, M. G. Lagally, S. G. Kruglik, and R. J. D. Miller, Electronically Driven Structure Changes of Si Captured by Femtosecond Electron Diffraction, Physical Review Letters , 4 (2008).

[15] A. M. Lindenberg, J. Larsson, K. Sokolowski-Tinten, K. J. Gaffney, C. Blome, O. Synnergren, J. Sheppard, C. Caleman, A. G. MacPhee, D. Weinstein, D. P. Lowney, T. K. Allison, T. Matthews, R. W. Falcone, A. L. Cavalieri, D. M. Fritz, S. H. Lee, P. H. Bucksbaum, D. A. Reis, J. Rudati, P. H. Fuoss, C. C. Kao, D. P. Siddons, R. Pahl, J. Als-Nielsen, S. Duesterer, R. Ischebeck, H. Schlarb, H. Schulte-Schrepping, T. Tschentscher, J. Schneider, D. von der Linde, O. Hignette, F. Sette, H. N. Chapman, R. W. Lee, T. N. Hansen, S. Techert, J. S. Wark, M. Bergh, G. Huldt, D. van der Spoel, N. Timneanu, J. Hajdu, R. A. Akre, E. Bong, P. Krejcik, J. Arthur, S. Brennan, K. Luening, and J. B. Hastings, Atomic-Scale Visualization of Inertial Dynamics, Science 308, 392 (2005)

[16] P. Stampfli and K. H. Bennemann, Time dependence of the laser-induced femtosecond lattice instability of Si and GaAs: Role of longitudinal optical distortions, Physical Review B 49, 7299 (1994), publisher: APS.

[17] E. S. Zijlstra, J. Walkenhorst, and M. E. Garcia, Anharmonic Noninertial Lattice Dynamics during Ultrafast Nonthermal Melting of InSb, Physical Review Letters 101, 135701 (2008), publisher: American Physical Society.

[18] K. Sokolowski-Tinten, C. Blome, J. Blums, A. Cavalleri, C. Dietrich, A. Tarasevitch, I. Uschmann, E. Förster, M. Kammler, M. Horn-von-Hoegen, and D. von der Linde, Femtosecond X-ray measurement of coherent lattice vibrations near the Lindemann stability limit, Nature (London) 422, 287 (2003)

[19] T. Zier, M. E. Garcia, and P. L. Silvestrelli, Comment on "Ab initio evidence for nonthermal characteristics in ultrafast laser melting", Phys. Rev. B 99, 066301 (2019).

[20] C. Lian, S. B. Zhang, and S. Meng, Reply to "Comment on "Ab initio evidence for nonthermal characteristics in ultrafast laser melting " ", Phys. Rev. B 99, 066302 (2019)

[21] D. M. Fritz, D. A. Reis, B. Adams, R. A. Akre, J. Arthur, C. Blome, P. H. Bucksbaum, A. L. Cavalieri, S. Engemann, and S. Fahy, Ultrafast bond softening in bismuth: Mapping a solid's interatomic potential with x-rays, Science 315, 633 (2007), publisher: American Association for the Advancement of Science.

[22] E. D. Murray, S. Fahy, D. Prendergast, T. Ogitsu, D. M. Fritz, and D. A. Reis, Phonon dispersion relations and softening in photoexcited bismuth from first principles, Physical Review B 75, 184301 (2007), publisher: APS.

[23] A. V. Kolobov, M. Krbal, P. Fons, J. Tominaga, and T. Uruga, Distortion-triggered loss of long-range order in solids with bonding energy hierarchy, Nature Chemistry 3, 311 (2011), number: 4 Publisher: Nature Publishing Group.

[24] R. A. Voronkov, N. Medvedev, and A. E. Volkov, Superionic State in Alumina Produced by Nonthermal Melting, physica status solidi (RRL) - Rapid Research Letters 14,
1900641 (2020)

[25] B. Y. Mueller and B. Rethfeld, Relaxation dynamics in laser-excited metals under nonequilibrium conditions, Physical Review B 87, 035139 (2013), publisher: APS.

[26] L. Waldecker, R. Bertoni, R. Ernstorfer, and J. Vorberger, Electron-Phonon Coupling and Energy Flow in a Simple Metal beyond the Two-Temperature Approximation, Physical Review X 6, 021003 (2016), arXiv:1507.03743 [cond-mat.mtrl-sci].

[27] Z. Lin and L. V. Zhigilei, Thermal excitation of d band electrons in $\mathrm{Au}$ : implications for laser-induced phase transformations, in High-Power Laser Ablation VI, Vol. 6261 (International Society for Optics and Photonics, 2006) p. 62610U.

[28] E. Bévillon, J.-P. Colombier, V. Recoules, and R. Stoian, First-principles calculations of heat capacities of ultrafast laser-excited electrons in metals, Applied Surface Science 336, 79 (2015), publisher: Elsevier.

[29] Z. Lin, L. V. Zhigilei, and V. Celli, Electron-phonon coupling and electron heat capacity of metals under conditions of strong electron-phonon nonequilibrium, Physical Review B 77, 075133 (2008), publisher: American Physical Society.

[30] G. V. Sin'ko, N. A. Smirnov, A. A. Ovechkin, P. R. Levashov, and K. V. Khishchenko, Thermodynamic functions of the heated electron subsystem in the field of cold nuclei, High Energy Density Physics 9, 309 (2013), publisher: Elsevier.

[31] S. L. Daraszewicz, Y. Giret, H. Tanimura, D. M. Duffy, A. L. Shluger, and K. Tanimura, Determination of the electron-phonon coupling constant in tungsten, Applied Physics Letters 105, 023112 (2014), publisher: American Institute of Physics.

[32] E. Bévillon, J.-P. Colombier, V. Recoules, and R. Stoian, Free-electron properties of metals under ultrafast laser-induced electron-phonon nonequilibrium: A firstprinciples study, Physical Review B 89, 115117 (2014), publisher: APS.

[33] F. Bottin and G. Zérah, Formation enthalpies of monovacancies in aluminum and gold under the condition of intense laser irradiation, Physical Review B 75, 174114 (2007), publisher: American Physical Society.

[34] N. A. Inogamov and Y. V. Petrov, Thermal conductivity of metals with hot electrons, Journal of Experimental and Theoretical Physics 110, 446 (2010), publisher: Springer.

[35] K. P. Migdal, Y. V. Petrov, and N. A. Inogamov, Kinetic coefficients for d-band metals in two-temperature states created by femtosecond laser irradiation, in Fundamentals of Laser-Assisted Micro-and Nanotechnologies 2013, Vol. 9065 (International Society for Optics and Photonics, 2013) p. 906503.

[36] B. J. Siwick, J. R. Dwyer, R. E. Jordan, and R. J. D. Miller, An Atomic-Level View of Melting Using Femtosecond Electron Diffraction, Science 302, 1382 (2003).

[37] B. J. Siwick, J. R. Dwyer, R. E. Jordan, and R. Miller, Femtosecond electron diffraction studies of strongly driven structural phase transitions, Chemical Physics 299, 285 (2004)

[38] M. Kandyla, T. Shih, and E. Mazur, Femtosecond dynamics of the laser-induced solid-to-liquid phase transition in aluminum, Physical Review B 75, 214107 (2007), publisher: American Physical Society.

[39] C. Guo, G. Rodriguez, A. Lobad, and A. J. Taylor, Structural Phase Transition of Aluminum Induced by 
Electronic Excitation, Physical Review Letters 84, 4493 (2000)

[40] V. Recoules, J. Clérouin, G. Zérah, P. M. Anglade, and S. Mazevet, Effect of Intense Laser Irradiation on the Lattice Stability of Semiconductors and Metals, Physical Review Letters 96, 055503 (2006).

[41] N. Jourdain, L. Lecherbourg, V. Recoules, P. Renaudin, and F. Dorchies, Ultrafast thermal melting in nonequilibrium warm dense copper, Phys. Rev. Lett. 126, 065001 (2021)

[42] M. Z. Mo, Z. Chen, R. K. Li, M. Dunning, B. B. L. Witte, J. K. Baldwin, L. B. Fletcher, J. B. Kim, A. Ng, R. Redmer, A. H. Reid, P. Shekhar, X. Z. Shen, M. Shen, K. Sokolowski-Tinten, Y. Y. Tsui, Y. Q. Wang, Q. Zheng, X. J. Wang, and S. H. Glenzer, Heterogeneous to homogeneous melting transition visualized with ultrafast electron diffraction, Science 360, 1451 (2018).

[43] S. L. Daraszewicz, Y. Giret, N. Naruse, Y. Murooka, J. Yang, D. M. Duffy, A. L. Shluger, and K. Tanimura, Structural dynamics of laser-irradiated gold nanofilms, Physical Review B 88, 184101 (2013), publisher: APS.

[44] R. Ernstorfer, M. Harb, C. T. Hebeisen, G. Sciaini, T. Dartigalongue, and R. J. D. Miller, The Formation of Warm Dense Matter: Experimental Evidence for Electronic Bond Hardening in Gold, Science 323, 1033 (2009), publisher: American Association for the Advancement of Science Section: Report.

[45] Y. Giret, S. L. Daraszewicz, D. M. Duffy, A. L. Shluger, and K. Tanimura, Nonthermal solid-to-solid phase transitions in tungsten, Physical Review B 90, 094103 (2014).

[46] S. T. Murphy, S. L. Daraszewicz, Y. Giret, M. Watkins, A. L. Shluger, K. Tanimura, and D. M. Duffy, Dynamical simulations of an electronically induced solid-solid phase transformation in tungsten, Physical Review B 92, 134110 (2015), publisher: APS.

[47] H. Zhang, C. Li, E. Bevillon, G. Cheng, J.-P. Colombier, and R. Stoian, Ultrafast destructuring of laser-irradiated tungsten: thermal or nonthermal process, Physical Review B 94, 224103 (2016), publisher: APS.

[48] Y. Tanaka and S. Tsuneyuki, Possible electronic entropydriven mechanism for non-thermal ablation of metals, Applied Physics Express 11, 046701 (2018).

[49] S. Feng and X. Cheng, First-principles investigation on metal tantalum under conditions of electronic excitation, Computational Materials Science 50, 3110 (2011).

[50] N. Medvedev and I. Milov, Nonthermal phase transitions in metals, Scientific Reports 10, 12775 (2020)

[51] X. Gonze, B. Amadon, P.-M. Anglade, J.-M. Beuken, F. Bottin, P. Boulanger, F. Bruneval, D. Caliste, R. Caracas, and M. Côté, ABINIT: First-principles approach to material and nanosystem properties, Computer Physics Communications 180, 2582 (2009), publisher: Elsevier.

[52] P. Hohenberg and W. Kohn, Inhomogeneous Electron Gas, Physical Review 136, B864 (1964), publisher: American Physical Society.

[53] W. Kohn and L. J. Sham, Self-Consistent Equations Including Exchange and Correlation Effects, Physical Re- view 140, A1133 (1965), publisher: American Physical Society.

[54] N. D. Mermin, Thermal Properties of the Inhomogeneous Electron Gas, Physical Review 137, A1441 (1965), publisher: American Physical Society.

[55] J. P. Perdew, K. Burke, and M. Ernzerhof, Generalized Gradient Approximation Made Simple, Physical Review Letters 77, 3865 (1996), publisher: American Physical Society.

[56] N. Troullier and J. Martins, A straightforward method for generating soft transferable pseudopotentials, Solid State Communications 74, 613 (1990), publisher: Elsevier.

[57] E. P. Silaeva, E. Bévillon, R. Stoian, and J.-P. Colombier, Ultrafast electron dynamics and orbital-dependent thermalization in photoexcited metals, Physical Review B 98, 094306 (2018), publisher: APS.

[58] H. J. Monkhorst and J. D. Pack, Special points for Brillouin-zone integrations, Physical Review B 13, 5188 (1976), publisher: American Physical Society.

[59] S. Feng and X. Cheng, First-principles investigation on metal tantalum under conditions of electronic excitation, Computational materials science 50, 3110 (2011).

[60] C. Kittel, Introduction to solid state physics, 8th ed. (J. Wiley, 2005).

[61] M. Volkov, S. A. Sato, F. Schlaepfer, L. Kasmi, N. Hartmann, M. Lucchini, L. Gallmann, A. Rubio, and U. Keller, Attosecond screening dynamics mediated by electron localization in transition metals, Nature Physics 15, 1145 (2019), publisher: Nature Publishing Group.

[62] Y. Cao and E. Conrad, Anomalous thermal expansion of ni (001), Physical review letters 65, 2808 (1990).

[63] N. Medvedev and I. Milov, Electron-phonon coupling in metals at high electronic temperatures, Physical Review B 102, 064302 (2020), publisher: APS.

[64] G. Grimvall, B. Magyari-Köpe, V. Ozoliņš, and K. A. Persson, Lattice instabilities in metallic elements, Reviews of Modern Physics 84, 945 (2012).

[65] X. Sedao, C. Maurice, F. Garrelie, J.-P. Colombier, S. Reynaud, R. Quey, and F. Pigeon, Influence of crystal orientation on the formation of femtosecond laserinduced periodic surface structures and lattice defects accumulation, Applied Physics Letters 104, 171605 (2014).

[66] X. Sedao, M. V. Shugaev, C. Wu, T. Douillard, C. Esnouf, C. Maurice, S. Reynaud, F. Pigeon, F. Garrelie, L. V. Zhigilei, et al., Growth twinning and generation of high-frequency surface nanostructures in ultrafast laserinduced transient melting and resolidification, ACS nano 10, 6995 (2016).

[67] E. Bévillon, J.-P. Colombier, and R. Stoian, Firstprinciples assessment of potential ultrafast laser-induced structural transition in Ni, Applied Surface Science 374, 365 (2016), publisher: Elsevier.

[68] S. R. Phillpot, S. Yip, and D. Wolf, How do crystals melt? computer simulations demonstrate the interplay between thermodynamics and kinetics during the melting process, Computers in physics 3, 20 (1989). 